\title{
Research Communication
}

\section{ApoE Polymorphism Is Associated With C-Reactive Protein in Low-HDL Family Members and in Normolipidemic Subjects}

\author{
Juhani Kahri, ${ }^{1}$ Aino Soro-Paavonen, ${ }^{1,2}$ Christian Ehnholm, ${ }^{3}$ and Marja-Riitta Taskinen ${ }^{1}$ \\ ${ }^{1}$ Division of Cardiology, Department of Internal Medicine, Helsinki University Central Hospital, Haartmaninkatu 4, \\ PO Box 340, 00029 HUS, Finland \\ ${ }^{2}$ Baker Medical Research Institute, Melbourne, Victoria, Australia \\ ${ }^{3}$ Department of Molecular Medicine, Biomedicum, National Public Health Institute, 00251 Helsinki, Finland
}

Received 30 December 2005; Revised 15 March 2006; Accepted 20 March 2006

\begin{abstract}
The study was aimed to compare inflammatory parameters between carriers of apoE4 isoforms (apoE4/3, apoE4/2, and apoE4/4 phenotypes) and those of carrying apoE3 isoform without apoE4 isoform (apoE3/3 phenotypes and apoE2/3 phenotypes). The concentrations of serum hsCRP, sVCAM-1, sICAM-1, and sE-selectin were measured in 211 subjects from Finnish low-HDL families and in 157 normolipidemic subjects. The subjects with apoE4 isoform had lower concentrations of serum hsCRP both in low-HDL family members $(p<0.05)$ and in normolipidemic subjects $(p<0.01)$. The differences in serum CRP values remained significant after adjustment for age, BMI, smoking status, hypertension, gender, lipoprotein variables, and family number. We conclude that apoE phenotype has a strong influence on serum CRP values.
\end{abstract}

Copyright ( $) 2006$ Juhani Kahri et al. This is an open access article distributed under the Creative Commons Attribution License, which permits unrestricted use, distribution, and reproduction in any medium, provided the original work is properly cited.

\section{INTRODUCTION}

A substantial body of evidence indicates, that low-grade inflammation in the vascular endothelium promotes atherogenesis $[1,2]$. This is indirectly evidenced by the fact that several inflammatory markers are linked to an increased risk of atherosclerosis. High-sensitive CRP (hsCRP) has been reported to associate with an increased risk of myocardial infarction, stroke, sudden death from cardiac causes, and peripheral arterial disease [3]. In addition, hsCRP has been shown to predict the first cardiovascular event better than low-density lipoprotein cholesterol (LDL-C) [3].

Recruitment of inflammatory cells from the circulation and their transendothelial migration is one of the earliest phases of atherogenesis [3]. Vascular endothelium expresses cellular adhesion molecules (CAMs) in response to several inflammatory stimuli [4]. The evidence for the role of CAMs in the pathogenesis of atherosclerosis has emerged from experimental studies which have demonstrated the focal expression of adhesion molecules in atherosclerotic plaques [4-6].

Different CAMs have various functions in the vasculature. Selectins and their ligands are involved in the first steps in leukocyte adhesion at sites of endothelial injury as they mediate the rolling and tethering of leukocytes in the vas- cular wall $[1,2]$. Intercellular adhesion molecule-1 (ICAM1) and vascular cell adhesion molecule-1 (VCAM-1) induce firm adhesion of inflammatory cells to the vascular surface [3]. Overall, both soluble ICAM-1 (sICAM-1) and soluble VCAM-1 (sVCAM-1) have been linked to increase risk of atherosclerosis [7-9].

Apolipoprotein E (apoE) is secreted from hepatocytes and macrophages. ApoE is transported in lipoproteins, and it regulates the hepatic uptake of remnant lipoproteins and facilitates cholesterol efflux from foam cells [10]. In addition, apoE has a central role in inflammation and in mediating the central nervous system response to injury [11-13]. There are three common ApoE isoforms, designated E2, E3, and E4, which are encoded for by distinct alleles on human chromosome 19. ApoE isoforms are distributed in six different phenotypes E2/2, E2/3, E3/3, E4/2, E4/3, and E4/4 [10]. The presence of $\mathrm{E} 4$ isoform is associated with coronary heart disease [14] and with the early onset of Alzheimer's disease [15].

So far, there are sparse data on apoE polymorphism in relation to the parameters of inflammation. Mänttäri et al [16] showed that dyslipidemic men with E4/4, E4/3, and E4/2 phenotypes exhibit lower CRP values than men carrying other apoE phenotypes. In a recent report by März et al [17], carriers of apoE2 allele and apoE3/3 homozygotes had significantly higher CRP than individuals with the genotypes 
apoE3/4 and apoE4/4. In dyslipidemic men and women, Creactive protein has been reported to be lower in subjects with one or two copies of E4 allele than in those with zero E4 allele [18]. Paradoxically, E4 allele has been associated with an enhanced inflammatory reaction after cardiopulmonary bypass [19]. In line, transgenic mice expressing the E4 allele had greater systemic and brain elevations of proinflammatory cytokines than E3 carriers [20].

We hypothesize that the apoE phenotype, as a critical component in inflammatory processes, may influence the levels of inflammation markers. HDL has been demonstrated to exhibit antiinflammatory properties by inhibiting cytokine induced expression of adhesion molecules [21$23]$. In contrast, acute inflammation process may decrease HDL concentration and thus compromise its antiatherogenic function $[24,25]$. Interestingly, recycling of apoE is closely linked to HDL metabolism [26]. Therefore we studied the effects of apoE phenotype on the levels of CRP and sCAMs on a sample of Finnish low-HDL families and in normolipidemic subjects.

\section{SUBJECTS AND METHODS}

The study subjects were recruited according to the study protocol as reported previously [27]. To identify the low-HDL families, first degree relatives of the probands were examined. The family members were coded as affected if their HDL-C levels were below the 10th percentile cut-off levels. If at least two affected subjects were identified in the family, all available second-degree relatives and spouses were contacted and included in the study. The blood samples for the lipid and lipoprotein analysis were taken at the same visit as the inflammation parameters and therefore the lipid and lipoprotein values in this study represent the values of that visit, and not those of the screening visit. Altogether 211 lowHDL family members and 157 normolipidemic subjects had measures of their apoE phenotype, hsCRP, and sVCAM-1 concentrations. Seventeen low-HDL subjects had history of CHD. The normolipidemic group comprised healthy volunteers and normolipidemic spouses from the low-HDL families. They were required to have no signs of $\mathrm{CHD}$ or diabetes, and no lipid lowering medication. The smoking status of the patients was categorized into current smokers and nonsmokers (including exsmokers). Hypertension was defined as self-reported use of antihypertensive drugs, systolic blood pressure $\geq 140 \mathrm{mmHg}$, or diastolic blood pressure $\geq$ $90 \mathrm{mmHg}$. Estrogen use was defined as any means of estrogen administration (oral contraceptives, or oral or transdermal postmenopausal hormone replacement therapy). Venous blood samples were collected after overnight fasting. Serum was separated and used for the biochemical measurements. Lipid and lipoprotein measurements were done from serum as described previously [27]. Serum levels of sICAM1, sVCAM-1, and sE-selectin were measured by using commercially available monoclonal antibody-based ELISAs ( $\mathrm{R}$ \& D Systems, Minneapolis, MN, USA). Serum highly sensitive CRP (hsCRP) was determined by a commercial kit from Medix Biochemica, Kauniainen, Finland. Subjects with
CRP levels over $10 \mathrm{mg} / \mathrm{l}$ were excluded. ApoE phenotype was determined by the method of Havekes et al [28]. Statistical comparisons of clinical and biochemical parameters were performed with SPSS 11.0 for Windows (SPSS Inc., Chigaco, IL, USA). Data are expressed as mean \pm standard error of mean (SEM). Continuous variables were compared between two groups by Student $t$ test. Variables with nonnormal distribution were $\log _{10}$-transformed before comparison. $P<.05$ was considered to be significant (two-tailed). The frequency distribution between the groups was compared with the Chisquare test. The correlation coefficients between the biochemical and clinical characteristics were examined by Pearson's correlation for continuous variables. Multiple regression analysis was performed to determine the relative contribution of different variables to hsCRP and sVCAM-1 values. In the low-HDL group, the subjects came from 47 different families. Ten families had only one subject, and five families had 11-16 subjects. Because the subjects in the present cohort were not independent, we used the family number (which indicates belonging to a certain family) as a random variable. Family-number-adjusted residuals of log CRP and $\log$ VCAM were used in the multiple regression model for low-HDL family members. Since control subjects were not related, the family-number adjustment was performed only in the low-HDL group.

\section{RESULTS}

The effects of apoE phenotype was studied by dividing subjects into two subgroups (apoE groups): into the E3 group, which included E3/2 and E3/3 phenotypes, and into the E4 group which included E4/2, E4/3, and E4/4 phenotypes. Clinical and biochemical characteristics of the study subjects are presented in Table 1.

Table 2 shows that serum hsCRP values were significantly lower in the E4 than in the E3 subgroup both in the low-HDL group $(P<.01)$ and in the normolipidemic group $(P<.05)$ after adjustment for age, BMI, serum apoA-I, apoA-II, apoB, HDL-C, LDL-C, triglycerides, smoking status, hypertension, and gender in normolipidemic subjects, and after additional adjustment for family number in the low-HDL group. The concentration of serum sVCAM-1 was significantly lower in the E4 group than in the E3 group in low-HDL subjects $(P<.01)$ but the significance disappeared after adjustment for family number (Table 2 ). Multiple regression analysis revealed that apoE phenotype was a significant predictor of serum hsCRP values both in low-HDL and normolipidemic subjects $(P=.023$ and $P=.011$, resp) (Table 3$)$. Estrogen used was also a strong predictor of serum hsCRP concentration in low-HDL subjects $(P=.026)$ as well as in normolpidemic subjects $(P=.036)$ (Table 3$)$.

\section{DISCUSSION}

The major finding of the present study is that subjects presenting the apoE4 allele have decreased levels of serum hsCRP. This is an interesting observation, since ApoE plays a central role in inflammation and atherosclerosis. ApoE 
TABLE 1: The clinical characteristics and lipid values of the study subjects.

\begin{tabular}{|c|c|c|c|c|}
\hline & \multicolumn{2}{|c|}{ Low-HDL subjects $n=211$} & \multicolumn{2}{|c|}{ Normolipidemic subjects $n=157$} \\
\hline & E3 group & E4 group & E3 group & E4 group \\
\hline & $N=140$ & $N=71$ & $N=101$ & $N=56$ \\
\hline $\operatorname{AGE}(y)$ & $41.5 \pm 1.2$ & $41.5 \pm 1.8$ & $51.8 \pm 1.1$ & $49.3 \pm 1.8$ \\
\hline BMI $(\mathrm{kg} / \mathrm{m} 2)$ & $25.6 \pm 0.3$ & $25.6 \pm 0.5$ & $24.9 \pm 0.4$ & $24.3 \pm 0.4$ \\
\hline Gender (male/female) & $68 / 72$ & $41 / 30$ & $45 / 56$ & $25 / 31$ \\
\hline Smokers (\%) & $17.1 \%$ & $31.0 \% *$ & $18.8 \%$ & $16.1 \%$ \\
\hline Hypertension(\%) & $27.1 \%$ & $22.5 \%$ & $27.7 \%$ & $17.9 \%$ \\
\hline Estrogen use(\%) & $17.1 \%$ & $18.3 \%$ & $15.8 \%$ & $19.6 \%$ \\
\hline Cholesterol (mmol/l) & $5.05 \pm 0.07$ & $5.27 \pm 0.13$ & $5.05 \pm 0.08$ & $5.13 \pm 0.11$ \\
\hline Triglycerides (mmol/l) & $1.32 \pm 0.06$ & $1.53 \pm 0.09$ & $1.05 \pm 0.08$ & $0.95 \pm 0.04$ \\
\hline HDL-cholesterol (mmol/l) & $1.30 \pm 0.04$ & $1.14 \pm 0.04^{*}$ & $1.51 \pm 0.04$ & $1.51 \pm 0.05$ \\
\hline LDL-cholesterol (mmol/l) & $3.15 \pm 0.08$ & $3.43 \pm 0.12$ & $3.07 \pm 0.07$ & $3.19 \pm 0.10$ \\
\hline ApoA-I (mg/dl) & $136 \pm 2$ & $129 \pm 2$ & $147 \pm 3$ & $145 \pm 3$ \\
\hline ApoA-II (mg/dl) & $36 \pm 1$ & $36 \pm 1$ & $37 \pm 1$ & $37 \pm 1$ \\
\hline ApoB (mg/dl) & $98 \pm 2$ & $106 \pm 3^{\ddagger}$ & $91 \pm 2$ & $91 \pm 2$ \\
\hline
\end{tabular}

Data are expressed as means \pm SEMs.

${ }^{*} P<.05,{ }^{\ddagger} P<.06$ after adjustment for smoking.

TABLE 2: The concentrations of hsCRP, sVCAM-1, sICAM-1, and sE-SELECTIN in Low-HDL subjects and normolipidemic subjects divided by apoE subgroups.

\begin{tabular}{lcccc}
\hline & \multicolumn{2}{c}{ Low-HDL subjects $n=211$} & \multicolumn{2}{c}{ Normolipidemic subjects $n=157$} \\
\hline ERP $(\mathrm{mg} / \mathrm{l})$ & $1.72 \pm 0.16$ & E4 group $n=71$ & E3 group $n=101$ & E4 group $n=56$ \\
sVCAM-1 $(\mathrm{ng} / \mathrm{ml})$ & $540 \pm 11$ & $1.14 \pm 0.17^{* *}$ & $1.29 \pm 0.14$ & $0.63 \pm 0.08^{*}$ \\
sICAM-1 $(\mathrm{ng} / \mathrm{ml})$ & $234 \pm 5$ & $478 \pm 12$ & $496 \pm 10$ & $494 \pm 13$ \\
sE-selectin $(\mathrm{ng} / \mathrm{ml})$ & $49 \pm 2$ & $238 \pm 7$ & $223 \pm 6$ & $236 \pm 5$ \\
\hline
\end{tabular}

Data are expressed as means \pm SEMs. ${ }^{* *} P<.01 ;{ }^{*} P<.05$ for the comparison with the E3 group after adjustment for age, gender, BMI, hypertension, smoking status, and lipoprotein variables; and family number in the low-HDL group.

TABLE 3: Multiple regression analysis of predictors of CRP values in low-HDL and normolipidemic subjects.

\begin{tabular}{|c|c|c|c|c|}
\hline \multirow[b]{2}{*}{ Variable } & \multicolumn{2}{|c|}{ Low-HDL subjects } & \multicolumn{2}{|c|}{ Normolipidemic subjects } \\
\hline & Standardized $\beta$ & $P$ & Standardized $\beta$ & $P$ \\
\hline Age & +0.147 & .080 & +0.244 & .003 \\
\hline BMI & +0.339 & $<.001$ & +0.315 & $<.001$ \\
\hline Smoking status & +0.129 & .057 & -0.027 & .703 \\
\hline Hypertension & +0.024 & .732 & +0.000 & .997 \\
\hline Gender & +0.092 & .230 & -0.154 & .878 \\
\hline LDL-C & -0.013 & .899 & +0.064 & .524 \\
\hline Triglycerides & +0.106 & .410 & +0.119 & .246 \\
\hline HDL-C & +0.082 & .620 & +0.204 & .173 \\
\hline ApoA-I & +0.008 & .961 & +0.260 & .062 \\
\hline ApoA-II & -0.086 & .384 & +0.062 & .473 \\
\hline ApoB & +0.057 & .676 & -0.031 & .789 \\
\hline Estrogen use & +0.166 & .026 & +0.161 & .036 \\
\hline ApoE grouping & -0.150 & .023 & -0.178 & .011 \\
\hline
\end{tabular}

The adjusted multiple $R^{2}$ is $0.202(P<.001)$ for low-HDL subjects and $0.326(P<.001)$ for normolipidemic subjects when all variables are included. The dependent variable in low-HDL family members is the family number adjusted residual of log CRP, and in normolipidemic subjects, the dependent variable is log CRP. Variables with skewed distribution were log-transformed before entering into the model. 
knockout mice are especially prone to atherosclerosis [10] and to die from lipopolysaccharide (LPS)-induced sepsis [11]. ApoE participates in cholesterol efflux, and has been observed to be recycled from triglyceride-rich lipoproteins into HDL-particles $[10,26]$. In mice, exogenous apoE injection has been demonstrated to decrease the LPS-induced production of proinflammatory cytokines, probable by decreasing the release of cytokines from macrophages, which was accompanied by reduced mortality [11]. CRP and apoE concentrations are inversely related in obese patients [12]. In addition, human CRP transgene expression has been shown to cause accelerated atherosclerosis in apolipoprotein E-deficient mice [13]. Thus, according to these studies, the role of apoE in inflammation and atherosclerosis may be protective.

Despite the importance of apoE in the inflammatory process, only few studies have linked apoE polymorphisms to inflammatory parameters. In the central nervous system, apoE4 has been reported to be less effective than apoE3 or apoE2 at suppressing the activation of microglia in cell culture models of brain inflammation [29]. Transgenic mice expressing the apoE4 allele have significantly greater systemic and brain elevations of proinflammatory cytokines TNF-alpha and IL-6 as compared with their apoE3 counterparts [20]. In the present study, the hsCRP concentration was significantly lower in subjects exhibiting apoE4 allele than in those exhibiting other alleles. Likewise, Mänttäri and associates demonstrated, in a population of 179 dyslipidemic Finnish men, that subjects with apoE4/3 phenotype had lower mean CRP concentration than those with apoE3/3 phenotype [16]. In Japanese Americans, CRP values have been reported to be significantly higher in apoE2 carriers than in apoE3 or apoE4 carriers, and similar between apoE3 and apoE4 carriers [30]. In line with the Japanese data, German patients with or without coronary heart disease also showed higher CRP values in apoE2 carriers than in apoE3/4 heterozygotes or in apoE4/4 homozygotes [17]. However, CRP was also significantly higher in apoE3/3 homozygotes than in apoE3/4 heterozygotes or in apoE4/4 homozygotes [17]. Similarly, in the Cardiovascular Health Study [31], CRP values were lower in apoE4 positive than in apoE4 negative individuals independently of the amount of used alcohol. Thus, the present data extend these previous observations to a cohort of low-HDL family members as well as to normolipidemic subjects.

The mechanisms behind low serum CRP values in apoE4 carriers in this study, and in previous works $[16-18,31]$ are unclear. Since apoE phenotype remained as a predictor of hsCRP in multiple regression model, the difference between apoE4 and apoE3 groups can not be explained by confounding factors, such as estrogen use. One explanation, originally suggested by März and associates [17], could be that the metabolism of CRP is related to mevalonate pathway. In carriers of apoE4, the mevalonate pathway may be downregulated resulting in decreased CRP [17].

The observed low serum CRP values in apoE4 carriers in the present study, and reportedly low CRP values in apoE4 carriers in previous works $[16-18,31]$, are perplexing find- ings since apoE4 allele is associated to an increased risk of atherosclerosis [14]. However, in the present study, the lowHDL family members within the E4 group had an atherogenic lipid profile: they had higher concentrations of apoB, a trend for higher serum triglycerides, and lower concentration of HDL-C as compared with the E3 group. Thus, the changes in lipoproteins rather than changes in inflammatory parameters may explain the atherogenity of apoE4 allele.

We conclude that apoE phenotype has a strong influence on C-reactive protein. ApoE may be a double-faced protein having different actions in lipid metabolism and inflammation.

\section{ACKNOWLEDGMENTS}

We thank all the study subjects for participating in this study. Virve Naatti, Helinä Perttunen-Nio, and Hannele Hilden are thanked for their excellent technical assistance. This work was supported by grants from the Finnish Cardiovascular Research Foundation, Special State Grants for health science research, The Finnish Medical Society Duodecim, The Sigrid Juselius Foundation, The International HDL Research Awards, The Paulo Foundation, Aarne Koskelo Foundation, Maud Kuistila Foundation, and The Biomedicum Helsinki Foundation.

\section{REFERENCES}

[1] Ross R. Atherosclerosis-an inflammatory disease. New England Journal of Medicine. 1999;340(2):115-126.

[2] Libby P, Ridker PM, Maseri A. Inflammation and atherosclerosis. Circulation. 2002;105(9):1135-1143.

[3] Ridker PM, Rifai N, Rose L, Buring JE, Cook NR. Comparison of C-reactive protein and low-density lipoprotein cholesterol levels in the prediction of first cardiovascular events. New England Journal of Medicine. 2002;347(20):1557-1565.

[4] Blankenberg S, Barbaux S, Tiret L. Adhesion molecules and atherosclerosis. Atherosclerosis. 2003;170(2):191-203.

[5] Davies MJ, Gordon JL, Gearing AJ, et al. The expression of the adhesion molecules ICAM-1, VCAM-1, PECAM, and Eselectin in human atherosclerosis. Journal of Pathology. 1993; 171(3):223-229.

[6] O’Brien KD, McDonald TO, Chait A, Allen MD, Alpers CE. Neovascular expression of E-selectin, intercellular adhesion molecule-1, and vascular cell adhesion molecule- 1 in human atherosclerosis and their relation to intimal leukocyte content. Circulation. 1996;93(4):672-682.

[7] Hwang SJ, Ballantyne CM, Sharrett RA, et al. Circulating adhesion molecules VCAM-1, ICAM-1 and E-selectin in carotid atherosclerosis and incident coronary heart disease cases. The atherosclerosis risk in communities (ARIC) study. Circulation. 1997;96(12):4219-4225.

[8] Ridker PM, Hennekens CH, Roitman-Johnson B, Stampfer MJ, Allen J. Plasma concentration of soluble intercellular adhesion molecule 1 and risks of future myocardial infarction in apparently healthy men. Lancet. 1998;351(9096):88-92.

[9] Rohde LE, Lee RT, Rivero J, et al. Circulating cell adhesion molecules are correlated with ultrasound-based assesment of carotid atherosclerosis. Arteriosclerosis, Thrombosis, and Vascular Biology. 1998;18(11):1765-1770. 
[10] Davignon J, Cohn JS, Mabile L, Bernier L. Apolipoprotein E and atherosclerosis: insight from animal and human studies. Clinica Chimica Acta. 1999;286(1-2):115-143.

[11] Van Oosten M, Rensen PCN, Van Amersfoort ES, et al. Apolipoprotein E protects against bacterial lipopolysaccharide-induced lethality. A new therapeutic approach to treat Gram-negative sepsis. Journal of Biological Chemistry. 2001; 276(12):8820-8824.

[12] Bach-Ngohou K, Nazih H, Nazih-Sanderson F, et al. Negative and independent influence of apolipoprotein $\mathrm{E}$ on C-reactive protein (CRP) concentration in obese adults. Potential antiinflammatory role of apoE in vivo. International Journal of Obesity. 2001;25(12):1752-1758.

[13] Paul A, Ko KWS, Li L, et al. C-reactive protein accelerates the progression of atherosclerosis in apolipoprotein E-deficient mice. Circulation. 2004;109(5):647-655.

[14] Song Y, Stampfer MJ, Liu S. Meta-analysis: apolipoprotein E genotypes and risk of coronary heart disease. Annals of Internal Medicine. 2004;141(2):137-147.

[15] Strittmatter WJ, Roses AD. Apolipoprotein E and Alzheimer's disease. Annual Review of Neuroscience. 1996;19:53-77.

[16] Mänttäri M, Manninen V, Palosuo T, Ehnholm C. Apolipoprotein E polymorphism and C-reactive protein in dyslipidemic middle aged men. Atherosclerosis. 2001;156(1):237-238.

[17] März W, Sxharnagl H, Hoffman MM, Boehm BO, Winkelmann BR. The apolipoprotein E polymorphism is associated with circulating C-reactive protein (the Ludwigshafen risk and cardiovascular health study). European Heart Journal. 2004;25 (23):2109-2119.

[18] Judson R, Brain C, Dain B, Windemuth A, Ruano G, Red C. New and confirmatory evidence of an association between APOE genotype and baseline C-reactive protein in dyslipidemic individuals. Atherosclerosis. 2004;177(2):345-351.

[19] Grocott HP, Newman MF, El-Moalem H, Bainbridge D, Butler A, Laskowitz DT. Apolipoprotein E genotype differentially influences the proinflammatory and anti-inflammatory response to cardiopulmonary bypass. Journal of Thoracic and Cardiovascular Surgery. 2001;122(3):622-623.

[20] Lynch JR, Tang W, Wang H, et al. ApoE genotype and an apoEmimetic peptide modify the systemic and central nervous system inflammatory response. Journal of Biological Chemistry. 2003;273(49):48529-48533.

[21] Cockerill GW, Rye KA, Gamble JR, Vadas MA, Barter PJ. Highdensity lipoprotein inhibit cytokine-induced expression of endothelial cell adhesion molecule. Arteriosclerosis, Thrombosis, and Vascular Biology. 1995;15(11):1987-1994.

[22] Cockerill GW, Huehns TY, Weerasinghe A, et al. Elevation of plasma high-density lipoprotein concentration reduces interleukin-1-induced expression of E-selectin in an in vivo model of acute inflammation. Circulation. 2001;103(1):108-112.

[23] Calabresi L, Franceschini G, Sirtori CR, et al. Inhibition of VCAM-1 expression in endothelial cells by reconstituted highdensity lipoproteins. Biochemical and Biophysical Research Communications. 1997;1238(1):61-65.

[24] Tietge UJF, Maugeais C, Cain W, et al. Overexpression of secretory phospholipase $\mathrm{A}(2)$ causes rapid catabolism and altered tissue uptake of high density lipoprotein cholesteryl ester and apolipoprotein A-I. Journal of Biological Chemistry. 1999;275 (14):10077-10084.

[25] Tietge UJF, Maugeais C, Lund-Katz S, Grass D, deBeer FC, Rader DJ. Human secretory phospholipase A2 (sPLA2) mediates decreased plasma levels of HDL-cholesterol and apoA-I in response to inflammation in human apoA-I transgenic mice.
Arteriosclerosis, Thrombosis, and Vascular Biology. 2002;22(7): 1213-1218.

[26] Heeren J, Grewal T, Laatsch A, et al. Recycling of apoprotein $\mathrm{E}$ is associated with cholesterol efflux and high density lipoprotein internalization. Journal of Biological Chemistry. 2003;278(16):14370-14378.

[27] Alagona C, Soro-Paavonen A, Ylitalo K, Salonen R, Salonen JT, Taskinen M-R. A low high density lipoprotein (HDL) level is associated with carotid artery intima-media thickness in asymptomatic members of low HDL families. Atherosclerosis. 2002;165(2):309-316.

[28] Havekes LM, de Knijff P, Beisiegel U, Havinga J, Smit M, Klasen E. A rapid micromethod for apolipoprotein E phenotyping directly in serum. Journal of Lipid Research. 1987;28(4): 455-463.

[29] Barger SW, Harmon AD. Microglial activation by Alzheimer amyloid precursor protein and modulation by apolipoprotein E. Nature. 1997;388(6645):878-881.

[30] Austin MA, Zhang C, Humphries SE, et al. Heritability of Creactive protein and association with apolipoprotein $\mathrm{E}$ genotypes in Japanese Americans. Annals of Human Genetics. 2004; 68(3):179-188.

[31] Mukamal KJ, Cushman M, Mittleman MA, Tracy RP, Siscovick DS. Alcohol consumption and inflammatory markers in older adults: the Cardiovascular Health Study. Atherosclerosis. 2004; 173(1):79-87. 


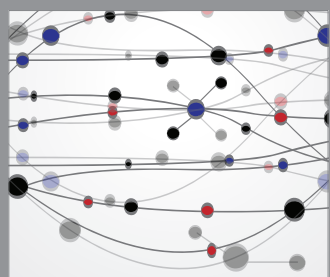

The Scientific World Journal
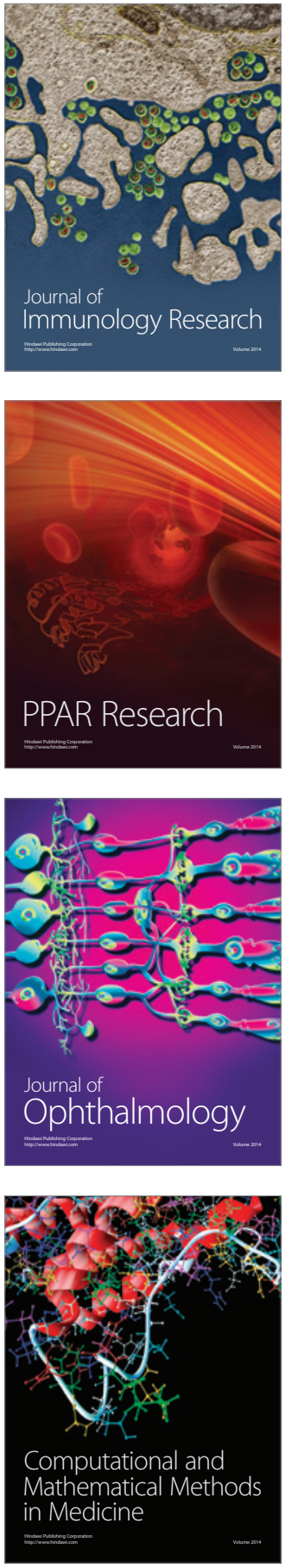

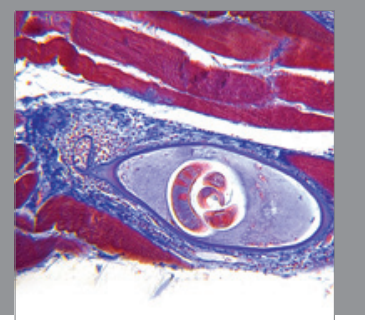

Gastroenterology

Research and Practice
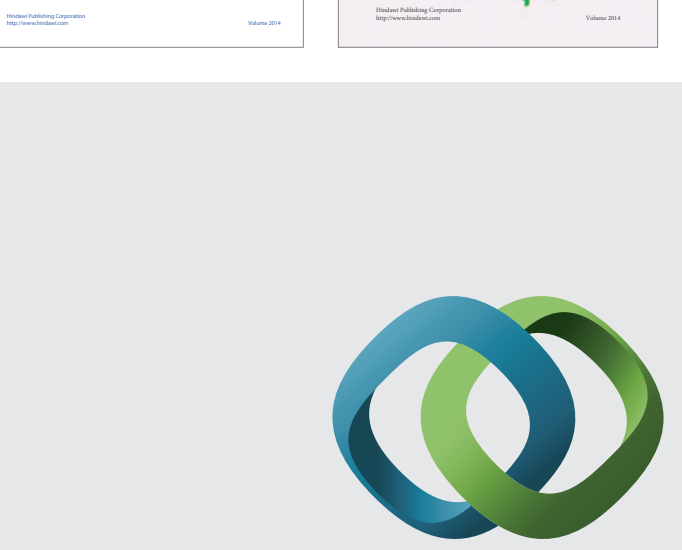

\section{Hindawi}

Submit your manuscripts at

http://www.hindawi.com
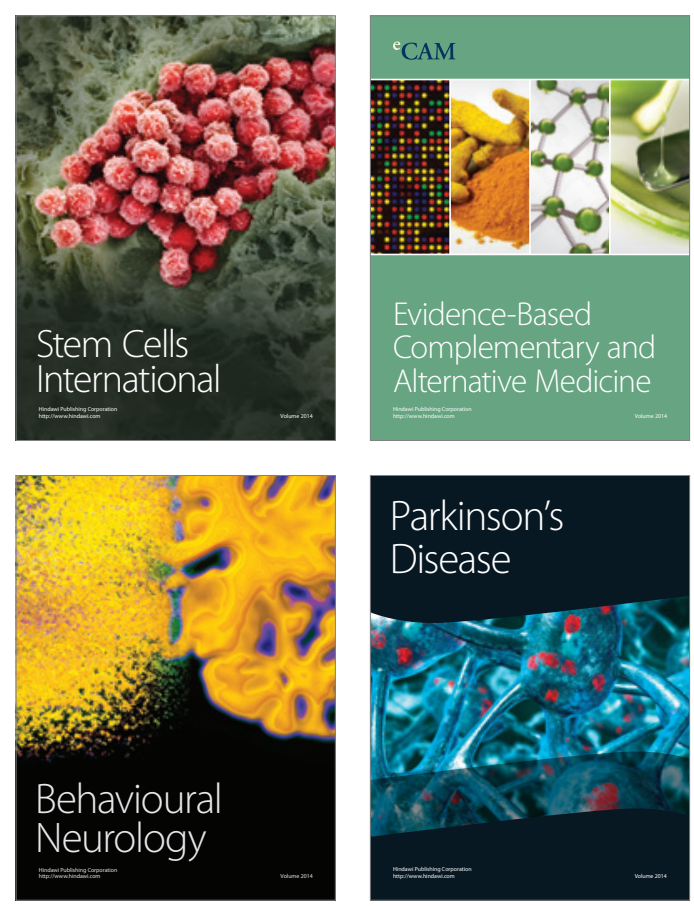

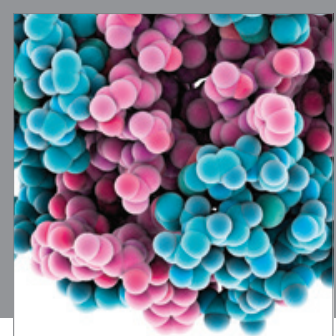

Journal of
Diabetes Research

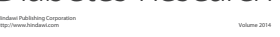

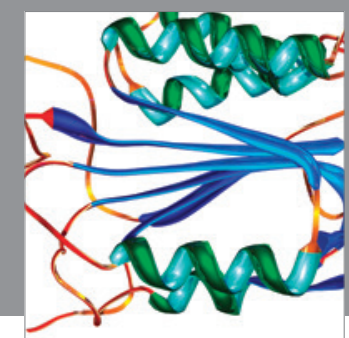

Disease Markers
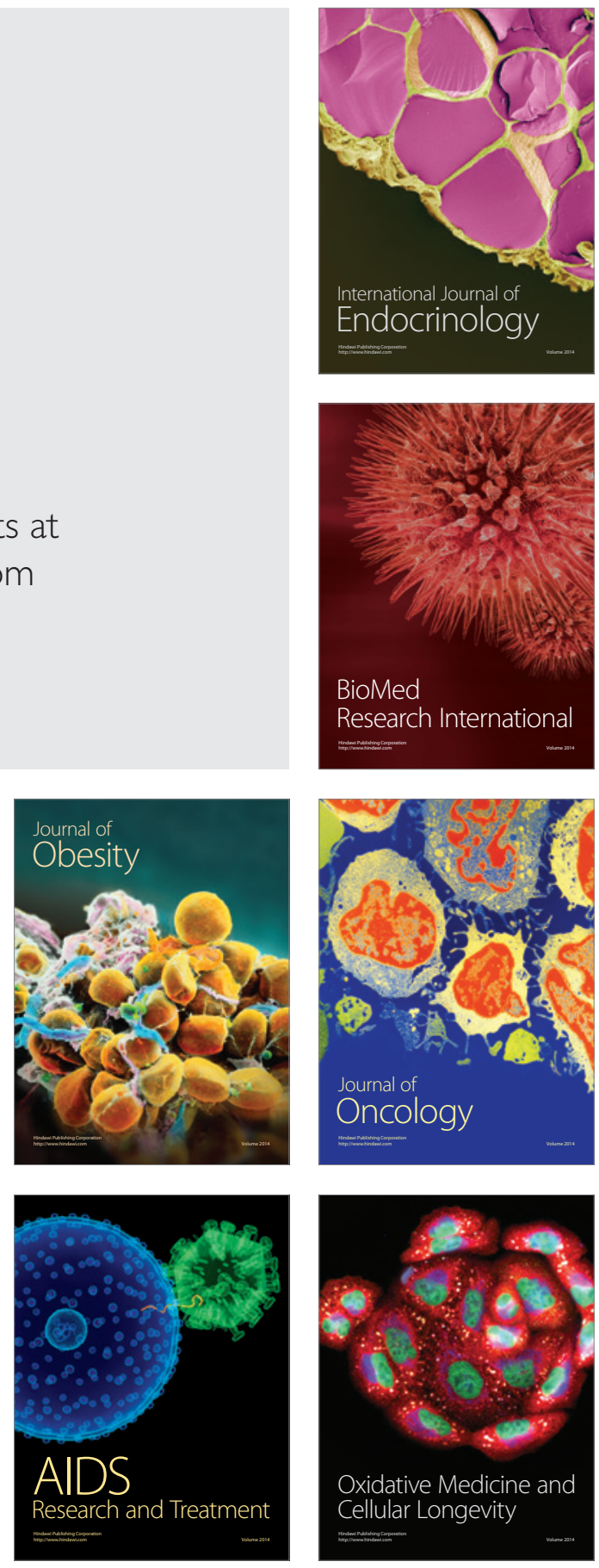